\title{
Universal upper bound for the entropy of superconducting vortices and the quantum Nernst effect
}

Cristina Diamantini

University of Perugia

Carlo Trugenberger

SwissScientific Technologies SA

Valerii Vinokur ( $\square$ vmvinokour@gmail.com )

Terra Quantum AG https://orcid.org/0000-0002-0977-3515

\section{Article}

Keywords: nernst, quantum, magnetic, effect

Posted Date: August 13th, 2021

DOl: https://doi.org/10.21203/rs.3.rs-789810/v1

License: (c) (i) This work is licensed under a Creative Commons Attribution 4.0 International License.

Read Full License

Version of Record: A version of this preprint was published at Quantum Reports on December 31st, 2021. See the published version at https://doi.org/10.3390/quantum4010002. 


\title{
Universal upper bound for the entropy of superconducting vortices and the quantum Nernst effect
}

\author{
M. C. Diamantini, ${ }^{1}$ C. A. Trugenberger,${ }^{2}$ \& V. M. Vinokur ${ }^{3,4, *}$
}

We show that the entropy per quantum vortex per layer in superconductors in external magnetic fields is bounded by the universal value $k_{\mathrm{B}} \ln 2$, which explains puzzling results of recent experiments on the Nernst effect. The observed plateau of the Nernst signal as a function of magnetic field is correspondingly attributed to a manifestation of the integer quantum Nernst effect.

The Nernst effect ${ }^{1,2}$ is the emergence of a transverse electric field produced by a longitudinal thermal gradient in the presence of a magnetic field and measures the flow of transverse entropy induced by a longitudinal particle motion. The Nernst effect has attracted a great attention after the discovery of a sizable Nernst coefficient in high-temperature cuprate superconductors due to fluctuating Cooper pairs and mobile vortices carrying simultaneously entropy and magnetic flux. The high interest in the Nernst effect is amplified by the fact that measurements of the Nernst signal can provide information about material parameters inaccessible by other means, for example, the upper critical field $H_{\mathrm{c} 2}$ that often cannot be directly measured because of its large value ${ }^{3}$. The Nernst signal has a maximum as a function of temperature and magnetic field and a tail into the normal state. Recent experiments ${ }^{4}$ revealed that, in the fluctuation region, the Nernst effect has the magnitude expected by theory. However, the peak amplitude of the Nernst signal is unexpectedly about the same in different superconductors despite the broad variance in basic superconducting parameters, and corresponds to the universal value of an entropy per vortex per layer $\approx k_{\mathrm{B}} \ln 2^{4}$.

Here we demonstrate that this remarkable finding is an immediate consequence of the dynamical symmetry of a superconducting ground state in an external magnetic field under the algebra $W_{1+\infty}$ of quantum area preserving the diffeomorphisms ${ }^{5-7}, \mathrm{see}^{8}$ for a review. The uniform ground states with a gap - like the Cooper pair condensate in superconductors - are incompressible in the limit of large gap, where density waves are suppressed. Note that although in a d-wave high- $T_{c}$ superconductor one could expect the gapless nodal modes to promote charge density waves (CDW), the maximum Nernst signal is observed in the region where superconductivity dominates ${ }^{4}$ and the competing CDW order does not develop. Hence Cooper condensate incompressibility is preserved there as well. The dynamical symmetry of the gapped systems (in the limit of large gap), classifying all possible configurations at the classical level, or ground states at the quantum level, is that of volume-preserving diffeomorphisms. In the presence of the applied external magnetic field the dynamical symmetry reduces to area-preserving diffeomorphisms in the plane orthogonal to the magnetic field.

This reduced case is particularly interesting since the algebra of areapreserving diffeomorphisms is a well-known extension of the 2D conformal algebra ${ }^{9}$. At the classical level, area-preserving diffeomorphisms are canonical transformations of a two-dimensional phase space. Normalizing all lengths by the magnetic length $\ell=\sqrt{\hbar c / e B}$, where $c$ is the light velocity, $-e$ is the elctron charge, and $B$ is the magnetic field, and endowing the plane with coordinates $z$ and $\bar{z}$ with a Poisson bracket

$$
\{f, g\}=i(\partial f \bar{\partial} g-\bar{\partial} f \partial g)
$$

one describes the area-preserving diffeomorphisms $\delta z=\{\mathcal{L}, z\}$ and $\delta \bar{z}=$ $\{\mathcal{L}, \bar{z}\}$ by generating functions $\mathcal{L}(z, \bar{z})$. The basis of generators $\mathcal{L}_{n, m}=$ $z^{n} \bar{z}^{m}$ satisfies the classical $w_{\infty}$ algebra ${ }^{8}$

$$
\left\{\mathcal{L}_{n, m}, \mathcal{L}_{k, l}\right\}=-i(m k-n l) \mathcal{L}_{n+k-1, m+l-1} .
$$

The operators $\mathcal{L}_{n m}$ with $n>0$ and $m>0$ form two closed sub-algebras, the two chiral sectors of the classical $w_{\infty}$ algebra, related by complex conjugation. Generators with both $n$ and $m$ negative, are descendants obtained as products of primary generators in the two fundamental chiral sectors. Finally, generators with $n=0$ and $m=0$ form two Abelian subalgebras.

The quantum version of this infinite-dimensional algebra is obtained by the usual substitution of Poisson brackets with quantum commutators: $i\{,\} \rightarrow[$,$] . Let us denote the quantum version of \mathcal{L}_{i-n, i}$ by $V_{n}^{i}$ and let us first discuss one single chiral sector of the quantum algebra by restricting to positive values of $i$. This gives the algebra $W_{\infty}$,

$$
\begin{aligned}
& {\left[V_{n}^{i}, V_{m}^{j}\right]=(j n-i m) V_{n+m}^{i+j-1}+q(i, j, n, m) V_{n+m}^{i+j-3}+\cdots} \\
& +\delta^{i j} \delta_{n+m, 0} c d(i, n),
\end{aligned}
$$

where the structure constants $q$ and $d$ are polynomials of their arguments and the dots denote a finite number of similar terms involving the operators $V_{n+m}^{i+j-1-2 k}$. The first term on the r.h.s. of Eq. (3) is the classical term (2). The remaining terms are quantum operator corrections, with the exception of the last c-number term, which represents a quantum anomaly with integer central charge $c$. All the quantum operator corrections are uniquely determined by the closure of the algebra; only the central charge $c$ is a free parameter. If we admit also the value $i=0$ we obtain the full algebra $W_{1+\infty}$, including the Abelian subalgebra quantum generators $V_{n}^{0}$. The quantization of the full classical algebra $w_{\infty}$, involving generators in the two chiral sectors, the two Abelian subalgebras, and all their products, is then obtained as the direct product of two copies $W_{1+\infty}$ and $\bar{W}_{1+\infty}$ of opposite chirality. If the chirality breaking external magnetic field is applied, only one of the two chiral sectors survive and the dynamical symmetry reduces to $W_{1+\infty}$. The integer central charge is a parameter of the effective field theory and distinguishes between different realizations of the incompressible quantum fluids. However, it is not important for our purposes, and, therefore, we will not further discuss it.

This infinite algebra has been applied to classify incompressible quantum fluids corresponding of the observed hierarchy of quantum Hall plateaus ${ }^{10-12}$, in which case the role of the vortices is taken by the Laughlin quasi-particles, with the same consequences described below. Not going into the details of the involved representation theory of the infinite-dimensional algebra ${ }^{13}$, we focus on the point crucial for present purposes, namely that the two generators $V_{-1}^{0}=z$ and $V_{1}^{1}=\bar{z}$ do not commute

$$
[z, \bar{z}]=\left[V_{-1}^{0}, V_{1}^{1}\right]=1 .
$$

Incompressibility leads to a phase-space reduction, the coordinate space itself becomes a phase space and the two coordinates do not commute.

${ }^{1}$ NiPS Laboratory, INFN and Dipartimento di Fisica e Geologia, University of Perugia, via A. Pascoli, I-06100 Perugia, Italy; ${ }^{2}$ SwissScientific Technologies SA, rue du Rhone 59, CH-1204 Geneva, Switzerland; ${ }^{3}$ Terra Quantum AG, St. Gallerstrasse 16A, CH-9400 Rorschach, Switzerland; ${ }^{4}$ City College of the City University of New York, 160 Convent Ave, New York, NY 10031, USA *Correspondence to: vmvinokour@gmail.com 
In the presence of a magnetic field this is tantamount to the well-known non-commutativity of the magnetic translations or of the guiding centres of charged particles in the first Landau level. Finite magnetic translations form nothing else than the group of area-preserving diffeomorphisms on the torus. Note, however that an external magnetic field is just one possible mechanism leading to an incompressible quantum fluid. Other mechanisms can exist that lead to the same non-commutativity as a consequence of their dynamical symmetry ${ }^{14}$.

Let us now focus on the consequences of this non-commutativity. We first recall that the standard example of a non-commutative space is phase space in quantum statistical mechanics. Restricting, for simplicity, to $1 \mathrm{D}$ systems we have a two-dimensional space with coordinates $(x, p)$ in which the momentum $p$ does not commute with the canonically conjugate variable $x$. As a consequence, phase space decomposes into fundamental cells of "area" $\hbar$ and each such cell contains maximally one fundamental quantum degree of freedom. This is how quantum degrees of freedom are counted. In our case, however, the non-commutativity between $z$ and $\bar{z}$ implies that it is the original two-dimensional, physical coordinate plane that behaves as a phase space at quantum level. As a consequence, there is a fundamental cell $A_{\text {fund }}$ in physical space which is the smallest area which can accommodate a quantum vortex degree of freedom. In superconductors, the fundamental excitations are vortices carrying one flux quantum $\Phi_{0}=\pi \hbar c / e$. Vortices carrying two or more flux quanta are simply superimposed fundamental excitations. The number of available configurations for maximally squeezed quantum vortices is thus $2^{N}$ with $N=A / A_{\text {fund }}$ the number of fundamental cells fitting in the sample. The maximum entropy of a quantum vortex per layer is thus the universal quantity $k_{\mathrm{B}} \ln 2$, in a full accord with the Nernst effect measurements ${ }^{4}$. This bound is simply a universal consequence of the incompressibility of a gapped, uniform ground state in a magnetic field and is common to all possible superconductors, independently of the details of their material parameters.

It has to be stressed that what we have just described is a property of quantum vortices. As soon as the applied magnetic field has created enough quantum vortices to completely fill up the available cells in a given sample, a further small increase of the magnetic field will not be able to accommodate more vortices. The observed plateau in the Nernst signal as a function of the magnetic field ${ }^{4}$ corresponds exactly to this integer quantum Nernst effect. It is possible that more sensitive experiments might detect a further plateau at half filling.

Finally, let us note that this result can be interpreted in terms of information theory. A maximally squeezed vortex is a fundamental bit. Therefore, a vortex "erased" from the sample by the transverse Nernst current takes away exactly the entropy $k_{\mathrm{B}} \ln 2$ according to the Landauer bound formula ${ }^{15}$.

\section{References}

1. Behnia, K. and Aubin, H. Nernst effect in metals and superconductors: a review of concepts and experiments. Rep. Progr. Phys. 79, 046502 (2016).

2. Varlamov, A. A., Galda, A.\& Glatz, A. Fluctuation spectroscopy: From RayleighJeans waves to Abrikosov vortex clusters. Rev. Mod. Phys. 90, 015009 (2018).

3. Yamashita, T., Shimoyama, Y., Haga, Y., Matsuda, T., Yamamoto, E., Onuki, Y., Sumiyoshi, H., Fujimoto, S., Levchenko, A., Shibauchi, T. \& Matsuda, Y. Colossal thermomagnetic response in the exotic superconductor $\mathrm{URu}_{2} \mathrm{Si}_{2}$. Nat. Phys. 11, 17 -20 (2015).

4. Rischau, C. W., Li, Y. Fauqué, B., Inoue, H., Kim, M., Bell, C., Hwang, H. Y., Kapitulnik, A., \& Behnia, K. Universal bound to the amplitude of the vortex Nernst signal in superconductors. Phys. Rev. Lett. 126, 077001 (2021).

5. Bakas, I. The large-N limit of extended conformal symmetries. Phys. Lett. B 228, 57 (1989).

6. Bakas, I. The structure of the $W_{\infty}$ algebra. Comm. Math. Phys. 134, 487 (1990).

7. Pope, C.N., Shen, X. \& Romans, J.L. $W_{\infty}$ and the Racah-Wigner algebra. Nucl. Phys. B 339 (1990).

8. Shen, X. $W_{\infty}$ and string theory. Int. J. Mod. Phys. A 7, 6953 (1990).

9. Francesco, P., Mathieu, P. \& Sènèchal, D. Conformal field theory. Springer-Verlag, New York (1997).

10. Cappelli, A., Trugenberger, C. A. \& Zemba, G. Infinite symmetry in the quantum Hall effect. Nucl. Phys. B 396, 465 (1993).

11. Cappelli, A., Trugenberger, C. A. \& Zemba, G. Classification of quantum Hall universality classes by $W_{1+\infty}$ symmetry. Phys. Rev. Lett. 71, 1969 (1993).

12. Cappelli, A., Trugenberger, C. A. \& Zemba, G. Stable hierarchical quantum Hall fluids as $W_{1+\infty}$ minimal models. Nucl. Phys. B 448, 470 (1995).

13. Kac, V. \& Radul, A. Quasifinite highest weight modules over the Lie algebra of differential operators on the circle. Comm. Math. Phys. 157, 429 (1993).

14. Diamantini M. C. \& Sodano, P. Characterizing topological order in superconducting systems. Phys. Rev. B 82, 144515 (2010).

15. Landauer, R. Irreversibility and heat generation in the computing process, IBM $J$. Res. Develop. 5, 183 (1961).

Acknowledgements The work by V.M.V. was supported by Terra Quantum and, partially, by the NSF grant DMR-1809188. M.C.D. thanks CERN, where she completed this work, for kind hospitality.

Author contribution M.C.D., C.A.T., and V.M.V. conceived the work, all the authors carried out calculations, discussed results, and wrote the paper.

Competing interests The authors declare that they have no competing interests.

Correspondence and requests for materials should be addressed to V.M.V. (vmvinokour@gmail.com). 